\title{
Too much of a good thing? Assessing performance tradeoffs of two-electron compounds for redox flow batteries
}

\author{
Bertrand J. Neyhouse, Alexis M. Fenton Jr., and Fikile R. Brushett* \\ Department of Chemical Engineering, Massachusetts Institute of Technology, Cambridge, MA 02139 \\ *Corresponding author: brushett@mit.edu
}

\begin{abstract}
Engineering redox-active compounds to support stable multi-electron transfer is an emerging strategy for enhancing the energy density and reducing the cost of redox flow batteries (RFBs). However, when sequential electron transfers occur at disparate redox potentials, increases in electrolyte capacity are accompanied by decreases in voltaic efficiency, restricting the viable design space. To understand these performance tradeoffs for two-electron compounds specifically, we apply theoretical models to investigate the influence of the electron transfer mechanism and redox-active species properties on galvanostatic processes. First, we model chronopotentiometry at a planar electrode to understand how the electrochemical response and associated concentration distributions depend on thermodynamic, kinetic, and mass transport factors. Second, using a zerodimensional galvanostatic charge / discharge model, we assess the effects of these key descriptors on performance for a single half-cell. Specifically, we examine how different properties (i.e., average of the two redox potentials, difference between the two redox potentials, charging rate, mass transfer rate, and comproportionation rate) affect the electrode polarization and voltaic efficiency. Finally, we extend the galvanostatic model to include two-electron compounds in both half-cells, demonstrating compounding voltage losses for a full cell. These results evince limitations to the applicability of multi-electron compounds - as such, we suggest new directions for molecular and systems engineering that may improve the prospects of these materials within RFBs.
\end{abstract}

Keywords: Redox flow battery, Multi-electron transfer, Electrochemical engineering, Zerodimensional modeling, Galvanostatic cycling, Chronopotentiometry 


\section{Introduction}

The development of low-cost redox couples and associated electrolytes is a promising costreduction pathway for redox flow batteries (RFBs). ${ }^{1,2}$ To this end, organic molecules and metalcoordination complexes are emerging as alternative charge storage species to compete with more traditional inorganic salts. These candidate materials are especially compelling for meeting production demands, as many can be derived from abundant, widely accessible sources and synthesized at-scale using existing process knowledge and infrastructure. ${ }^{3}$ Further, their chemical structures can be functionalized to refine key properties, such as the redox potential, electrochemical reversibility, solubility, and stability. ${ }^{4-6}$ Of particular note is the possibility for these materials to support multiple electron transfers, which, in principle, may facilitate marked increases in capacity and concomitant decreases in energy-specific electrolyte cost. ${ }^{7}$ However, multi-valent redox couples are often chemically irreversible due to the increasing instability and/or decreasing solubility of the charged species. ${ }^{8}$ Recent research efforts have led to the advancement of several multi-electron transfer molecular platforms, which have been tuned to mitigate major decomposition pathways and promote solubility across different oxidation states in relevant electrolytes, furthering their viability in practical embodiments. ${ }^{9-12}$ Despite this progress, the overall impact of the multi-electron approach on cell performance has not been widely explored beyond proof-of-concept demonstrations. ${ }^{7}$

While many molecular engineering challenges (e.g., stability, solubility, etc.) are universal to the design of redox electrolytes, the unique electron transfer mechanisms (sequential vs. concerted) of multi-electron compounds present a characteristic feature that may constrain their design. For example, many redox-active organic molecules that undergo multi-electron transfer in aqueous electrolytes (e.g., quinones, ${ }^{13-15}$ phenothiazines, ${ }^{16}$ and phenazines $^{17}$ ) typically exhibit multiple 
redox reactions occurring at similar potentials due to hydrogen bonding interactions present in these environments. ${ }^{18}$ Conversely, similar molecules used in non-aqueous electrolytes (e.g., phenothiazines, ${ }^{9,10}$ phenazines, ${ }^{19}$ and viologens ${ }^{20,21}$ ), some used in aqueous electrolytes (e.g., viologens ${ }^{22-24}$ ), and metal-coordination complexes containing non-innocent ligands ${ }^{11,25,26}$ often feature sequential electron transfer events with disparate and easily discernable redox potentials. Compared to the concerted mechanism, which presents minimal voltage losses (vide infra), the sequential mechanism imposes significant losses, increasing with the potential difference between redox events. Indeed, prior experimental work has shown that, during galvanostatic cell cycling, there is an asymmetry between voltage-time plateaus during charge and discharge: during the charging step, more time is spent at the higher cell voltage, while during the discharging step, more time is spent at the lower cell voltage..$^{9-12}$ This augments the difference between the average charge and discharge voltages, lowering both the voltaic and energy efficiencies of the cell. To assess the efficacy of multi-electron compounds, it is necessary to understand the magnitude of these losses, which, in turn, requires a deeper understanding of the extent to which the molecular properties and cell operating conditions contribute to RFB performance.

Low-dimensional reaction-transport models hold utility for uncovering the sources of performance loss and quantifying their contributions in electrochemical cells. Specifically, zerodimensional models, which are the central focus of this work, consider only time-dependent behavior, foregoing spatial variation and geometric constraints in the electrochemical and fluid dynamic properties to provide theoretically concise and computationally light modeling frameworks. ${ }^{27}$ Importantly, these simple analytical treatments-based on fundamental electrochemical engineering principles - enable the determination of tradeoffs in device performance for redox electrolytes prior to embarking on intensive cell cycling and modeling 
studies to understand complex behavior in geometrically-accurate domains. Such models have previously been used to assess crossover effects and various operating conditions in vanadium RFBs $^{28-31}$ and are beginning to be used to evaluate other candidate RFB platforms. ${ }^{32}$

In this work, we develop three electrochemical models (Figure 1) to understand the effect of two-electron compounds on cell cycling and assess their performance tradeoffs in RFBs. To gain initial insight into the underlying physics driving multi-electron transfer, we begin by analyzing the chronopotentiometric response of a one-dimensional planar electrode (Figure 1a), highlighting the influence of thermodynamic, kinetic, and mass transport factors on electrode polarization. Subsequently, using a single half-cell (Figure 1b) to simulate galvanostatic charge / discharge cycling, we show that voltaic efficiency is significantly affected by both the average redox potential and the potential difference between the redox events. To a lesser extent, different mass transfer rates between species, along with comproportionation reaction rates, further alter the cycling behavior. Finally, using a full cell galvanostatic cycling model (Figure 1c), we consider the impact of utilizing two-electron compounds in both half-cells, which results in compounding inefficiencies due to additional voltage losses and charge imbalance. By connecting molecular properties to cell performance, we are able to propose design criteria for more efficient highcapacity redox electrolytes. More broadly, this approach provides a framework for evaluating the impact of material properties on cell performance, which can be extended to investigate additional sources of inefficiency (e.g., activation and ohmic overpotentials) and other characteristic failure modes (e.g., molecular decomposition, crossover, etc.) for candidate systems. 
(a)

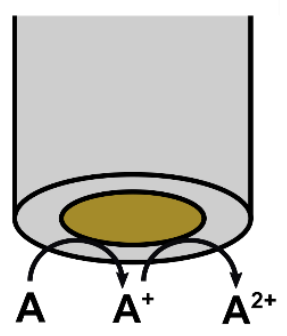

(b)

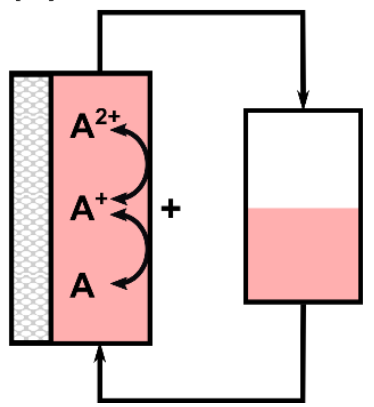

(c)

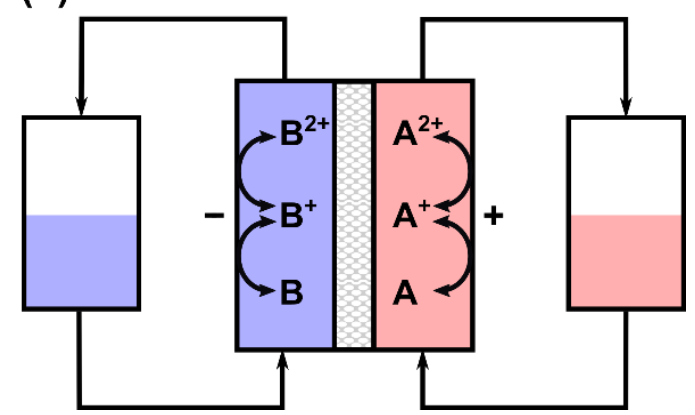

Figure 1. Schematic of modeling domains for (a) chronopotentiometry at a planar electrode, (b) galvanostatic charging / discharging for a single half-cell with a two-electron compound $A$, and (c) galvanostatic charging / discharging of a full cell with the two-electron compounds $A$ and $B$.

\section{Theoretical Framework}

The theoretical framework presented here describes concerted and sequential two-electron transfer processes by quantifying the voltage-time relationship typical of galvanostatic processes. Specifically, the sequential two-electron transfer for species $A$ is described by Equations (1) and (2).

$$
\begin{gathered}
A \rightleftharpoons A^{+}+e^{-} \\
A^{+} \rightleftharpoons A^{2+}+e^{-}
\end{gathered}
$$

In addition to heterogeneous electron transfer on the electrode surface, homogeneous comproportionation and disproportionation (Equation (3)) in the bulk must be considered when the redox potential of Equation (2) is more positive than that of Equation (1):

$$
A+A^{2+} \rightleftharpoons 2 A^{+}
$$

In contrast to Equations (1)-(3), species $A$ may undergo a concerted two-electron transfer in which the oxidation of $A$ proceeds directly to $A^{2+}$ according to Equation (4).

$$
A \rightleftharpoons A^{2+}+2 e^{-}
$$


In the following, Sections 2.1-2.3 discuss the role of thermodynamics, kinetics, and mass transport effects on the reactions in Equations (1)-(4), and Section 2.4 describes model nondimensionalization and execution.

\subsection{Thermodynamics and reaction kinetics}

To simplify the reaction kinetics and the overall theoretical analysis, all electrode reactions are assumed to be electrochemically reversible such that reactive species at the electrode surface are in equilibrium and their concentrations are governed by the Nernst Equation (Equations (5) and (6) for the first and second oxidation, respectively). We note this assumption is in agreement with prior experimental literature for many of the organic molecules and metal-coordination complexes under consideration for use in RFBs. ${ }^{2,5,7}$

$$
\begin{aligned}
& E=E_{1}^{(A)}+\frac{R T}{F} \ln \left(\frac{C_{A^{+}}^{s}}{C_{A}^{s}}\right) \\
& E=E_{2}^{(A)}+\frac{R T}{F} \ln \left(\frac{C_{A^{2+}}^{s}}{C_{A^{+}}^{s}}\right)
\end{aligned}
$$

In Equations (5) and (6), $E(\mathrm{~V})$ is the potential at the electrode surface, $E_{1}^{(A)}(\mathrm{V})$ is the formal redox potential of the first oxidation (Equation (1)), $E_{2}^{(A)}(\mathrm{V})$ is the formal redox potential of the second oxidation (Equation (2)), $R\left(8.314 \mathrm{~J} \mathrm{~mol}^{-1} \mathrm{~K}^{-1}\right)$ is the universal gas constant, $T(\mathrm{~K})$ is the absolute temperature (here, $T=298 \mathrm{~K}), F\left(96485 \mathrm{C} \mathrm{mol}^{-1}\right.$ ) is the Faraday constant, and $C_{j}^{s}$ (mol $\mathrm{m}^{-3}$ ) is the concentration of species $j$ at the electrode surface. It is also convenient to introduce $E_{\text {avg }}^{(A)}$ as the arithmetic mean of $E_{1}^{(A)}$ and $E_{2}^{(A)}$. Note that throughout this work, all potentials are defined relative to an arbitrary reference electrode. 
The rate of bulk comproportionation, $r_{p}\left(\mathrm{~mol} \mathrm{~m}^{-3} \mathrm{~s}^{-1}\right)$, and bulk disproportionation, $r_{d}\left(\mathrm{~mol} \mathrm{~m}^{-}\right.$ ${ }^{3} \mathrm{~s}^{-1}$ ) are given by Equations (7) and (8), respectively:

$$
\begin{aligned}
& r_{p}=k_{p} C_{A}^{\infty} C_{A^{+}}^{\infty} \\
& r_{d}=k_{d}\left(C_{A^{+}}^{\infty}\right)^{2}
\end{aligned}
$$

Where $\left.C_{j}^{\infty}(\mathrm{mol} \mathrm{m})^{-3}\right)$ is the concentration of species $j$ in the bulk solution and $k_{p}$ and $k_{d}\left(\mathrm{~m}^{3} \mathrm{~mol}^{-1}\right.$ $\mathrm{s}^{-1}$ ) are the comproportionation and disproportionation rate constants, respectively. These rate constants are related by the difference in formal redox potentials, $\Delta E^{(A)}=E_{2}^{(A)}-E_{1}^{(A)}$, according to Equation (9):

$$
k_{p}=k_{d} \exp \left(\frac{F}{R T} \Delta E^{(A)}\right)
$$

For a concerted two-electron transfer (Equation (4)), equilibrium concentrations at the electrode surface are again described by the Nernst equation as shown in Equation (10).

$$
E=E_{\text {avg }}^{(A)}+\frac{R T}{2 F} \ln \left(\frac{C_{A^{2+}}^{s}}{C_{A}^{s}}\right)
$$

Because the concerted step does not proceed through an intermediate, comproportionation and disproportionation are assumed to be negligible.

\subsection{Chronopotentiometry at a planar electrode}

To analyze the time-dependent potential response for sequential two-electron transfer at a planar electrode (Figure 1a), a one-dimensional model can be derived from the mass conservation equation in the absence of convection and migration (Equation (11)):

$$
\frac{\partial C_{j}}{\partial t}=-D \nabla^{2} C_{j}=-D \frac{\partial^{2} C_{j}}{\partial x^{2}}
$$


Subject to the following initial and boundary conditions:

$$
\begin{gathered}
C_{j}(t=0, x)=C_{j}^{\infty} \\
-D \frac{\partial C_{j}(t, x=0)}{\partial x}=N_{j} \\
C_{j}(t, x=\infty)=C_{j}^{\infty}
\end{gathered}
$$

Where $t(\mathrm{~s})$ is the time, $x(\mathrm{~m})$ is the axial position perpendicular to the planar electrode surface, $D$ $\left(\mathrm{m}^{2} \mathrm{~s}^{-1}\right)$ is the diffusivity, which is assumed to be the same for all species, $C_{j}\left(\mathrm{~mol} \mathrm{~m}^{-3}\right)$ is the local concentration of species $j$, and $N_{j}\left(\mathrm{~mol} \mathrm{~m}^{-2} \mathrm{~s}^{-1}\right)$ is the molar flux of species $j$ to the electrode surface. The solution to Equations (11)-(14) for the time-dependent concentration of species $j$ is given by Equation (15). ${ }^{33}$

$$
C_{j}(x, t)=C_{j}^{\infty}-\frac{1}{(\pi D)^{1 / 2}} \int_{0}^{t} \frac{-N_{j}(\eta)}{(t-\eta)^{1 / 2}} \exp \left(\frac{-x^{2}}{4 D(t-\eta)}\right) d \eta
$$

Note that $\eta$ is an integration variable. To derive the electrode potential as a function of time, Equation (16) relates the applied current, $I(\mathrm{~A})$, to the partial currents, $I_{1}$ and $I_{2}(\mathrm{~A})$, for the reactions given in Equations (1) and (2), respectively:

$$
I=I_{1}+I_{2}
$$

The partial currents are equivalent to the surface fluxes according to Equations (17)-(19):

$$
\begin{gathered}
N_{A}=-\frac{I_{1}}{F S} \\
N_{A^{+}}=\frac{I_{1}}{F S}-\frac{I_{2}}{F S} \\
N_{A^{2+}}=\frac{I_{2}}{F S}
\end{gathered}
$$


Here, $S\left(\mathrm{~m}^{2}\right)$ is the electrode surface area. Equation (15) can be combined with Equations (16)(19) for each species to determine the electrode potential as a function of time using Equations (5) and (6). These coupled, nonlinear equations can then be solved implicitly, as discussed in

\section{Section 2.4.}

\subsection{Galvanostatic cell cycling}

To describe the effects of sequential two-electron transfers on galvanostatic charge / discharge, a zero-dimensional model is derived (Figure 1b). Similar to the planar electrode case, the model applies a constant charging current (Equation (16)), which is the sum of the partial currents. Electrode kinetics are again described by Equations (5) and (6), and the relationship between the bulk and surface concentrations of each species can be described by convective mass transfer (Equations (20) and (21)) and the flux balance (Equation (22)):

$$
\begin{gathered}
I_{1}=F S k_{m, A}\left(C_{A}^{\infty}-C_{A}^{s}\right) \\
I_{2}=-F S k_{m, A^{2+}}\left(C_{A^{2+}}^{\infty}-C_{A^{2+}}^{s}\right) \\
I_{1}-I_{2}=-F S k_{m, A^{+}}\left(C_{A^{+}}^{\infty}-C_{A^{+}}^{s}\right)
\end{gathered}
$$

Here, $k_{m, j}\left(\mathrm{~m} \mathrm{~s}^{-1}\right)$ is the mass transfer coefficient of species $j$. Equations (20)-(22) can be combined with the equilibrium expressions in Equations (5) and (6) to arrive at an expression for the surface concentration of $A^{+}\left(C_{A^{+}}^{s}\right)$ :

$$
C_{A^{+}}^{s}=\frac{k_{m, A} C_{A}^{\infty}+k_{m, A^{+}} C_{A^{+}}^{\infty}+k_{m, A^{2+}} C_{A^{2+}}^{\infty}}{k_{m, A} \exp \left(\frac{F}{R T}\left(E_{1}^{(A)}-E\right)\right)+k_{m, A^{+}}+k_{m, A^{2+}} \exp \left(\frac{F}{R T}\left(E-E_{2}^{(A)}\right)\right)}
$$


The surface concentration is then substituted into Equation (16) to solve for the electrode potential as a function of the time-dependent bulk concentrations (Equation (24)). Again, the coupled nonlinear algebraic equations (Equations (23) and (24)) can be solved implicitly (vide infra).

$$
I=F S k_{m, A}\left[C_{A}^{\infty}-C_{A^{+}}^{s} \exp \left(\frac{F}{R T}\left(E_{1}^{(A)}-E\right)\right)\right]-F S k_{m, A^{2+}}\left[C_{A^{++}}^{\infty}-C_{A^{+}}^{s} \exp \left(\frac{F}{R T}\left(E-E_{2}^{(A)}\right)\right)\right]
$$

Finally, to track changes in the bulk concentration, the system is assumed to be well-mixed such that the total current is uniformly distributed throughout the entire volume. Note that the model treats the electrode half-cell, reservoir, and connecting tubing as a single continuous domain. For this treatment, the mass balances on the reactor volume for each species are a system of ordinary differential equations as shown by Equations (25)-(28),

$$
\begin{gathered}
V \frac{d C_{A}^{\infty}}{d t}=-\frac{I_{1}}{F}-V k_{p} C_{A}^{\infty} C_{A^{2+}}^{\infty}+V k_{d}\left(C_{A^{+}}^{\infty}\right)^{2} \\
V \frac{d C_{A^{+}}^{\infty}}{d t}=\frac{I_{1}}{F}-\frac{I_{2}}{F}+2 V k_{p} C_{A}^{\infty} C_{A^{2+}}^{\infty}-2 V k_{d}\left(C_{A^{+}}^{\infty}\right)^{2} \\
V \frac{d C_{A^{2+}}^{\infty}}{d t}=\frac{I_{2}}{F}-V k_{p} C_{A}^{\infty} C_{A^{2+}}^{\infty}+V k_{d}\left(C_{A^{+}}^{\infty}\right)^{2}
\end{gathered}
$$

which are subject to the initial conditions:

$$
C_{j}^{\infty}(t=0)=C_{j}^{o}
$$

$V\left(\mathrm{~m}^{3}\right)$ is the total electrolyte volume and $C_{j}^{o}\left(\mathrm{~mol} \mathrm{~m}^{-3}\right)$ is the initial bulk concentration. These coupled ordinary differential equations can be numerically solved to yield changes in bulk concentrations as a function of time, which can be further used to implicitly solve for the electrode potential (Equations (23) and (24)). 
For concerted electron transfer in the absence of comproportionation, the mass balances can be solved analytically, yielding time-dependent bulk concentrations and the subsequent electrode potential according to Equations (29)-(31):

$$
\begin{gathered}
C_{A}^{\infty}=C_{A}^{o}-\frac{I t}{2 F V} \\
C_{A^{2+}}^{\infty}=C_{A^{2+}}^{o}+\frac{I t}{2 F V} \\
E=E_{\text {avg }}^{(A)}+\frac{R T}{2 F} \ln \left(\frac{C_{A^{2+}}^{\infty}+\frac{I}{2 F S k_{m, A^{2+}}}}{C_{A}^{\infty}-\frac{I}{2 F S k_{m, A}}}\right)
\end{gathered}
$$

\subsection{Dimensionless variables and analysis}

To reduce the total number of independent model parameters, dimensionless variables are introduced. Equation (32) defines the dimensionless concentration, $\theta_{j}^{m}$, which describes the concentration in state $z(z \in\{s, \infty, o\})$ normalized by the total bulk concentration, $C_{T}^{\infty}\left(\mathrm{mol} \mathrm{m}^{-3}\right)$, which remains constant:

$$
\theta_{j}^{z}=\frac{C_{j}^{z}}{C_{A}^{\infty}+C_{A^{+}}^{\infty}+C_{A^{2+}}^{\infty}}=\frac{C_{j}^{z}}{C_{T}^{\infty}}
$$

Equation (33) defines the dimensionless charging current, $\Psi$, which is derived by normalizing the total charging current by the mass transfer limiting current for the oxidation of $A$ :

$$
\Psi=\frac{I}{F k_{m, A} S C_{T}^{\infty}}
$$

Similarly, Equation (34) defines a dimensionless time, $\tau$, which normalizes the cycle time by the theoretical charging time for one electron: 


$$
\tau=\frac{t I}{F V C_{T}^{\infty}}
$$

The mass transfer coefficients are normalized by that of species $A$ according to Equation (35) to yield dimensionless mass transfer coefficients, $\gamma_{j}$ :

$$
\gamma_{j}=\frac{k_{m, j}}{k_{m, A}}
$$

Finally, Equation (36) scales the rate of comproportionation by the charging current to give a dimensionless rate constant, $\kappa$ :

$$
\kappa=\frac{V k_{p}\left(C_{T}^{\infty}\right)^{2} F}{I}
$$

Note that for the discharging step, the current reverses sign, resulting in negative values for $\Psi$, $\tau$, and $\kappa$. To ensure the solutions comprise a consistent time domain $(\tau \in[0,2])$, the sign of $\tau$ is reversed. The complete dimensionless equations are provided in the Supplementary Information (SI).

In this analysis, the coupled ODEs (Equations (25)-(28)) were solved using the ode15s function in $\mathrm{MATLAB}^{\circledR} \mathrm{R} 2018 \mathrm{a}$, while nonlinear algebraic equations were solved implicitly using the fsolve function in the same program. Simulations were performed on a Dell Latitude 7290 laptop computer with an Intel ${ }^{\circledR}$ Core $^{\mathrm{TM}}$ i7-8650U processor (quad-core, $1.90 \mathrm{GHz}$ ) and randomaccess memory of 16 GB. Solving each charge / discharge cycle took approximately $20-30$ seconds. To allow for capacity stabilization during cycling, a total of five complete charge / discharge cycles were simulated (Figures S1 and S2) and results for the fifth cycle are reported, unless stated otherwise. 


\section{Results and Discussion}

\subsection{Chronopotentiometry at a planar electrode}

To gain insight into the underlying physics of electron transfer in two-electron compounds, we first model this process for a one-dimensional planar electrode (Figure 1a). Chronopotentiometry is a constant-current technique that measures potential at the electrode surface arising from surface redox reactions coupled with boundary layer diffusion, as opposed to galvanostatic cycling, where transport primarily takes place via the convection of bulk species whose concentrations change over time. Although the modes of transport are different, chronopotentiometry displays analogous behavior to galvanostatic cycling and can therefore serve as a basis for interpreting the underlying physics present in both systems. For brevity, we examine only the oxidation of $A$ to $A^{2+}$ via sequential electron transfers, assuming that all species have equal diffusion coefficients and comproportionation reaction rates are negligible. When a constant current is applied to a planar electrode, the reactant species concentration at the surface decreases until inevitably approaching zero, which results in a sharp increase in potential. The time required for this process is commonly referred to as Sand's time, ${ }^{34} t_{\text {sand }}^{(n)}$ (s), as shown in Equation (37):

$$
t_{\text {sand }}^{(n)}=\frac{\left(n F S C_{j}^{\infty}\right)^{2} \pi D_{j}}{4 I^{2}}
$$

$n$ is the number of electrons transferred per redox reaction (here, $n=1$ ). Figure 2a shows the timedependent potential response for a constant current applied to a planar electrode for a two-electron donating species, $A$, with an exemplary value of $\Delta E^{(A)}=0.6 \mathrm{~V}$. The first plateau, corresponding to the oxidation of $A$ to $A^{+}$(Equation (1)), is equivalent to a traditional Sand's time measurement for a one-electron transfer. Then, as $A^{+}$continues to be oxidized to $A^{2+}$ (Equation (2)), a much longer second plateau can be observed until eventually reaching another sharp increase in 
potential. Note that the time required to reach this second asymptote is equivalent to that for a concerted two-electron transfer (Equation (37)); however, similar to galvanostatic cycling, the time spent at each plateau is unequal. ${ }^{9-12}$

Although initially dissimilar plateau durations may seem unintuitive, a closer look at the temporal evolution of the concentration and current distributions reveals this to be a sensible outcome (Figures 2b and 2c). Consistent with conventional Sand's time, the concentration of species $A$ reaches zero at $t=t_{\text {sand }}^{(1)}$. However, as current continues to flow, $A$ does not stop diffusing from the bulk; rather, the oxidation of $A$ continues contributing significantly to the current, resulting in an elongation of its concentration profile. Correspondingly, the concentration of $A^{+}$at the surface (Figure 2c) increases sharply as $t \rightarrow t_{\text {sand }}^{(1)}$ but decreases more gradually because the partial current $\left(I_{2}\right)$ of the second oxidation remains low as $A^{+}$freely diffuses away from the electrode. Thus, despite the higher potential plateau corresponding to the second oxidation, the first oxidation continues to supply a significant fraction of current $\left(I_{1}\right)$, extending the duration of the second plateau as compared to the first. Although analytically simpler, this illustration of chronopotentiometric reaction-diffusion at a planar electrode is analogous to the physics present during galvanostatic charge / discharge and underpins the combined effects of thermodynamics, kinetics, and mass transfer on two-electron transfer compounds. 

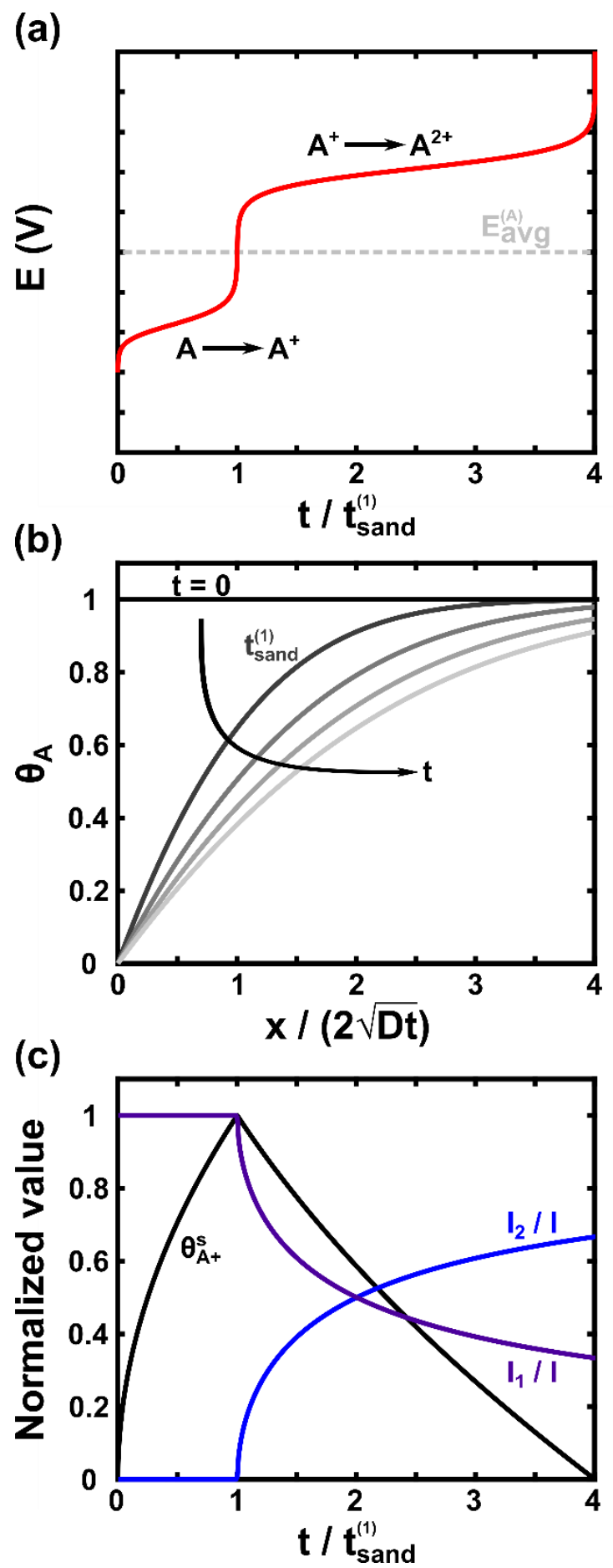

Figure 2. (a) Chronopotentiometry at a planar electrode for a generic $E_{a v g}^{(A)}$ and $\Delta E^{(A)}$; the response is independent of the applied current. (b) Temporal evolution of the dimensionless concentration profile for species $A$. (c) Temporal evolution of the dimensionless surface concentration of species $A^{+}$and the normalized partial currents. 


\subsection{Two-electron galvanostatic half-cell cycling}

With a foundational understanding of the combined factors influencing electrode polarization, we extended our analysis to galvanostatic charging / discharging of two-electron compounds to interrogate their cycling performance. Here, we consider only a single half-cell (Figure 1b), assuming the other half-cell remains at a fixed reference potential of $0 \mathrm{~V}$, and apply a constant, dimensionless charging rate for both charge and discharge $(\Psi)$, which is defined as the applied current relative to the mass transfer limiting current. The resulting potential is a measure of the kinetic and mass transport losses in the half-cell, but note that this model neglects ohmic losses. While these are generally a dominant source of voltage loss in RFBs, ${ }^{35}$ they are mostly independent of redox-active material properties, which are the focus of this work. To aid comparison, we initially assume all species have equal mass transfer coefficients $\left(\gamma_{A^{+}}=\gamma_{A^{2+}}=1\right)$ and that comproportionation reaction rates are negligible $(\kappa=0)$; these complicating factors will be introduced subsequently and sequentially to explore their individual effects.

Figure 3a shows a typical charge / discharge profile for a two-electron transfer process at an intermediate dimensionless current ( $\Psi=0.25)$, comparing the response of a concerted electron transfer to that of the more commonly observed sequential electron transfer both with and without a potential difference $\left(\Delta E^{(A)}=0.6 \mathrm{~V}\right.$ and $\Delta E^{(A)}=0 \mathrm{~V}$, respectively). Because $\tau$ is nondimensionalized by the charging capacity for a one-electron transfer, the theoretical capacity for the two-electron system is, by definition, $\tau=2$. Initially, we observe a non-negligible difference between the concerted and sequential $\left(\Delta E^{(A)}=0 \mathrm{~V}\right)$ electron transfer mechanisms. Specifically, there is a slightly larger difference between average charging and discharging potentials calculated for the sequential mechanism $(31.3 \mathrm{mV})$ compared to the concerted mechanism $(19.2 \mathrm{mV})$, which can be understood by considering that the sequential reaction proceeds through an intermediate, 
imposing additional mass transfer losses (and thus overpotential). When a potential difference is applied between the redox events, we observe the formation of two separate plateaus during cycling - during charging, the lower plateau corresponds to the oxidation of $A$ to $A^{+}$(Equations (1)) and the higher plateau corresponds to the oxidation of $A^{+}$to $A^{2+}$ (Equation (2)). For very small currents $(\Psi \approx 0.001)$, the plateaus are nearly symmetric (i.e., each plateau accounts for almost the same dimensionless time), and the theoretical capacity is accessed. However, as currents increase, the accessible capacity reduces and significant plateau asymmetry grows to the point where, at high enough currents, only one plateau is observed (Figure 3b). Like the planar electrode case (Figure 2), species $A$ is not consumed entirely once the second plateau is reached and continues contributing to the current, resulting in a slower consumption of $A^{+}$than if no $A$ remained. At dimensionless currents above $\Psi=0.5$, the charging profile displays a disappearance in the first plateau — at this point, the oxidation of $A$ on charge and the reduction of $A^{2+}$ on discharge are mass transfer limiting (zero surface concentration) at all points during cycling, meaning the electrode polarization is driven entirely by the second reaction step. Also, because the mass transfer coefficients are assumed to be equal, the resulting charge and discharge curves are symmetric; however, this changes under varying values of $\gamma_{A^{+}}$and $\gamma_{A^{2+}}$ (vide infra), as mass transfer overpotentials contribute unequally to charge and discharge. 
(a)

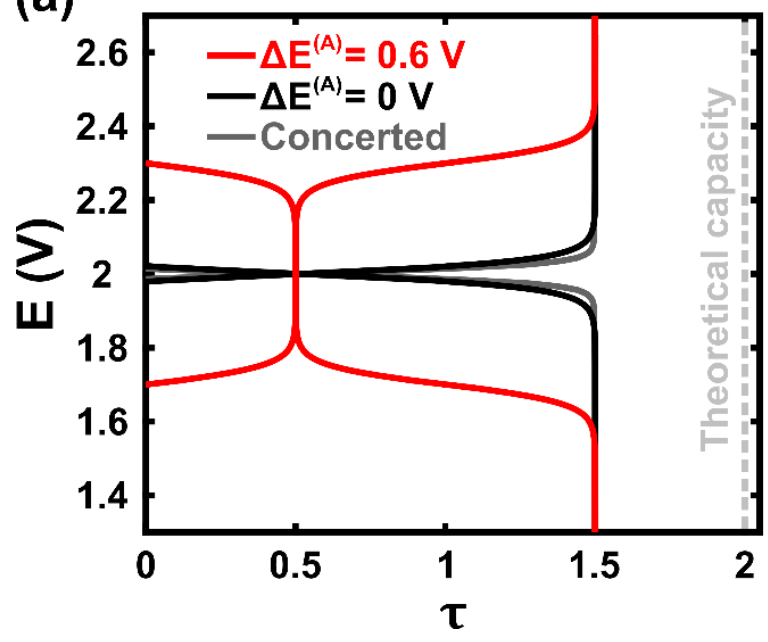

(b)

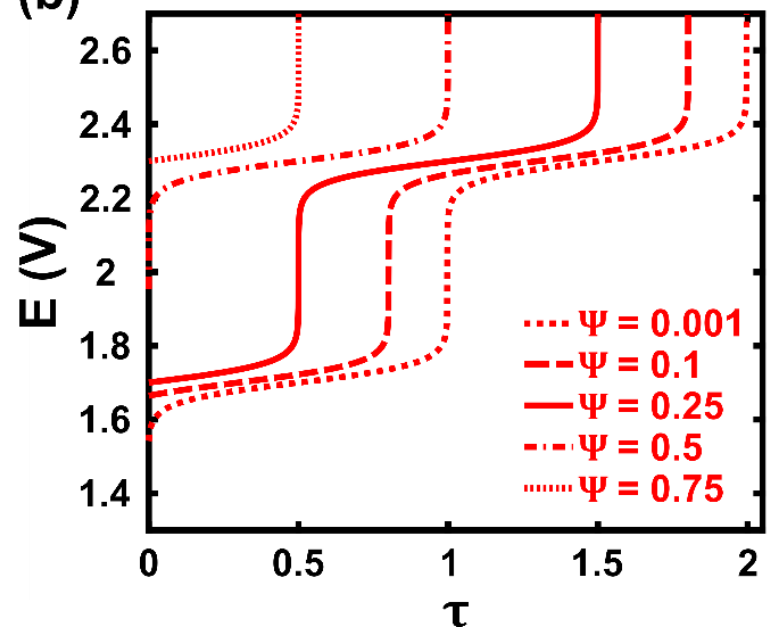

Figure 3. (a) Charge / discharge profiles shown for $\Psi=0.25$ and different values of the potential difference (sequential mechanism) compared to the concerted mechanism, and (b) charge profiles for increasing values of dimensionless charging current $\left(\Delta E^{(A)}=0.6 \mathrm{~V}\right)$. Results shown for $E_{\text {avg }}^{(A)}=2 \mathrm{~V}, \gamma_{A^{+}}=\gamma_{A^{2+}}=1$, and $\kappa=0$.

While there are several means by which the effects of asymmetry may be quantified, its most significant influence is on voltaic efficiency, which can be expressed as the ratio of the average discharge voltage to the average charge voltage. For reference, under typical cycling conditions, the coulombic efficiency is near unity, thus the voltaic efficiency is a reasonable approximation of the energy efficiency, defined as the product of the coulombic and voltaic efficiencies. In general, the voltaic efficiency decreases with increasing current as the effects of ohmic, kinetic, and mass transport losses become more pronounced, although as mentioned above, we neglect ohmic losses in this study. In addition, we consider losses from only one half-cell with reversible kinetics, and therefore, these values should be considered as upper bounds for the voltaic efficiency under the specified conditions. 
Figure 4 shows the combined effect of dimensionless charging current, potential difference, and average redox potential on the voltaic efficiency. The curves are characterized by two distinct regions - at low currents, the voltaic efficiency drops rapidly with increasing current due to an imbalance between the two plateaus; at higher currents, where the first plateau disappears completely, the voltaic efficiency slope parallels that of the case where $\Delta E^{(A)}=0 \mathrm{~V}$ with an offset in magnitude. Importantly, the presence of a potential difference between redox events drastically reduces voltaic efficiency under all conditions, though especially at increasing currents and lower average redox potentials, which are particularly relevant for aqueous chemistries. ${ }^{36}$ In general, voltage losses can be compensated with higher average redox potentials, but the voltaic efficiency for sequential processes may still suffer a 10 - 20\% decrease at moderate currents and high cell voltages compared to the concerted process, which represents the maximum voltaic efficiency. These losses in voltaic efficiency are substantial, especially considering they only account for one electrochemically reversible half-cell—additional kinetic (e.g., quasireversible and irreversible behavior, ${ }^{37}$ low electrochemically active surface area ${ }^{38}$ ) and ohmic losses (e.g., electrolyte and membrane resistance ${ }^{35}$ ) will further reduce overall efficiency.

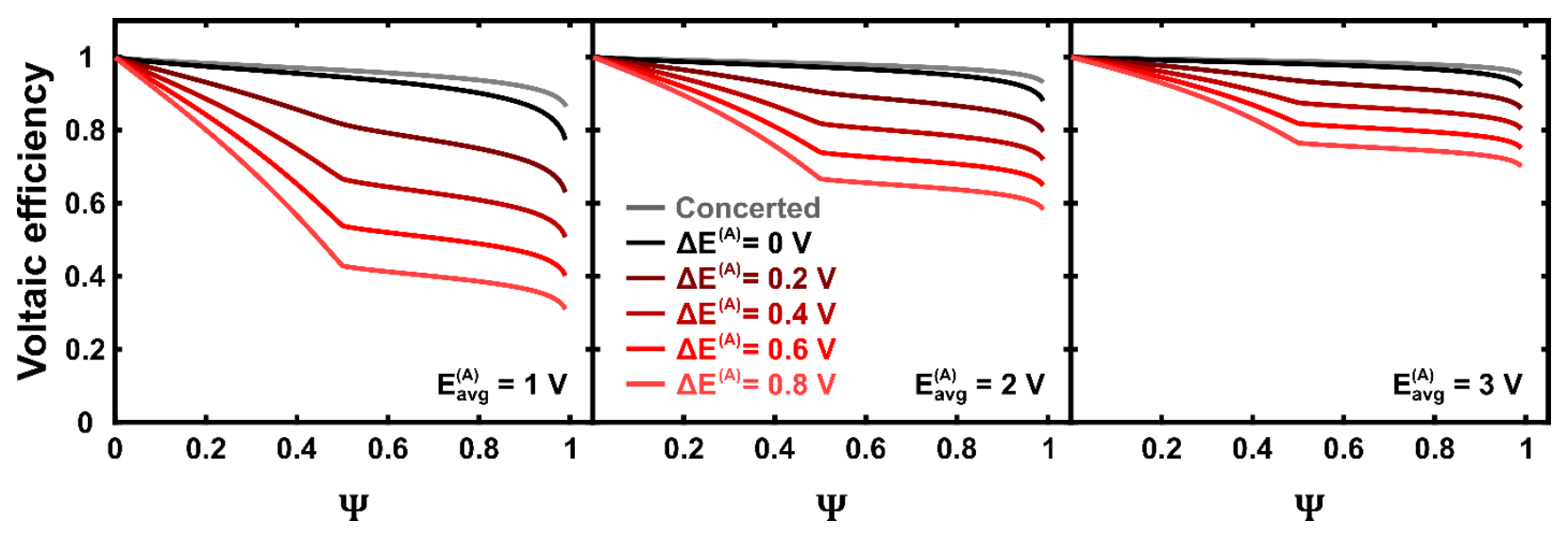


Figure 4. Voltaic efficiency as a function of dimensionless charging current, comparing a concerted two-electron transfer to a sequential two-electron transfer with varied $\Delta E^{(A)}$ for increasing values of $E_{\text {avg }}^{(A)}$. Results shown for $\gamma_{A^{+}}=\gamma_{A^{2+}}=1$ and $\kappa=0$.

Additional mass transfer losses can be evaluated by relaxing the assumption that all species have equal mass transfer coefficients; to simplify our analysis, we will consider cases where the mass transfer rates of $A^{+}$and $A^{2+}$ are equal $\left(\gamma_{A^{+}}=\gamma_{A^{2+}}\right)$ but differ from $A$. The values used here $\left(\gamma_{A^{+}}=0.8,1.2\right)$ were estimated based on previously reported Sherwood number correlations for $\mathrm{RFBs}^{39}$ for typical changes in diffusivity for soluble two-electron redox species in differing oxidation states. ${ }^{9,11}$ Although the variations in mass transfer are relatively small, the effects can be readily observed from the respective charge / discharge profiles (Figure 5a); specifically, the charging and discharging curves become asymmetric, as differing mass transfer rates affect the relative fluxes and, consequently, the surface concentrations of each species. Similar to the asymmetry between plateaus described already (see Figure 4), this additional asymmetry impacts the voltaic efficiency (Figure 5b). First, we observe the appearance of an additional change in slope at intermediate dimensionless currents corresponding to the disappearance of a single charge / discharge plateau. For example, when $\gamma_{A^{+}}=0.8$, the first plateau on the discharging curve will disappear while the first plateau on the charging curve persists. Second, as expected, slower mass transfer rates (e.g., reduced flow rates, higher electrolyte viscosity, larger redox-active molecules) correspond to lower voltaic efficiencies at all current values. 
(a)

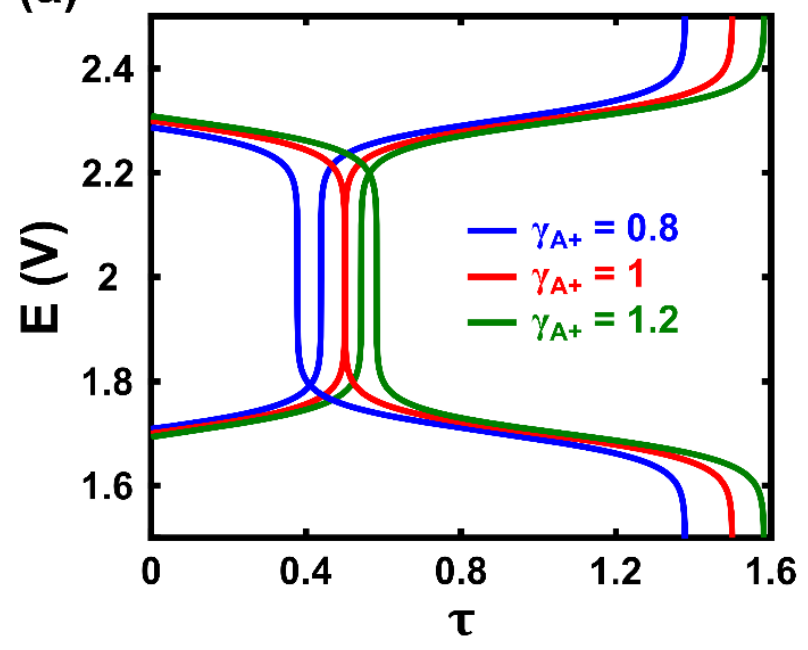

(b)

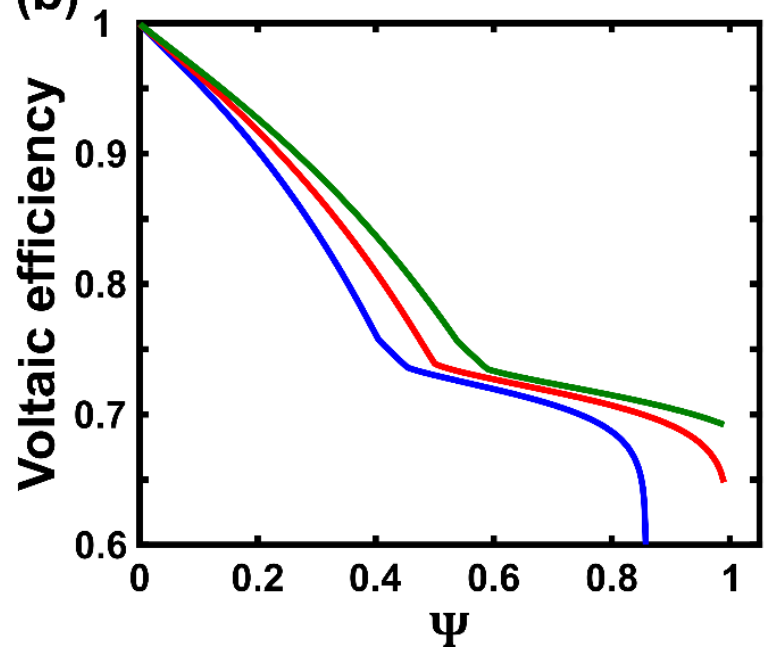

Figure 5. (a) Charge / discharge profiles shown for $\Psi=0.25$ and (b) voltaic efficiency as a function of dimensionless current for varying mass transfer coefficients $\left(\gamma_{A^{+}}=\gamma_{A^{2+}}\right)$. Results shown for $E_{\text {avg }}^{(A)}=2 \mathrm{~V}, \Delta E^{(A)}=0.6 \mathrm{~V}$, and $\kappa=0$.

Given the inclusion of variable mass transfer coefficients, the differences between individual species thus necessitates the treatment of comproportionation reactions. When considering sequential electrode reactions of identical kinetic and mass transport conditions, comproportionation does not play a distinguishable role in determining the electrode potential as the species identity does not influence boundary layer fluxes. ${ }^{40,41}$ Comproportionation yields a shift in the time-dependent bulk concentrations toward the formation of $A^{+}$(Figure 6a), and with varying mass transport coefficients, this causes charge / discharge curves to become increasingly asymmetric, thus accentuating voltaic efficiency losses that arise from differences in mass transfer (Figure 6b). However, for comproportionation to proceed, both $A$ and $A^{2+}$ must be present in solution concurrently - therefore, this effect only occurs where mass transfer limitations lead to simultaneous oxidation of $A$ and $A^{+}$(charging) or reduction of $A^{2+}$ and $A^{+}$(discharging). These simultaneous reactions only constitute a fraction of the total charging time, which increases with 
increasing current, meaning that its influence on voltaic efficiency only becomes discernable at dimensionless currents above $\Psi \approx 0.5$. Operation under such low accessible capacity regimes is likely to be impractical and inefficient, and as such, we tentatively conclude that comproportionation for two-electron transfer is negligible under typical galvanostatic conditions. Note that in this treatment, comproportionation and disproportionation reactions are assumed to occur only in the bulk as opposed to the mass transfer boundary layer. This assumption ignores the expected non-linear boundary layer concentrations, although even if the concentrations were to change in the boundary layer, the system is still beholden to the fact that comproportionation reactions only occur alongside the simultaneous oxidation / reduction of both species, so any significant effects will still be restricted to higher currents. As a result, we expect this will have only minor effects on the overall voltaic efficiency and, consequently, the conclusions drawn here are expected to remain valid.
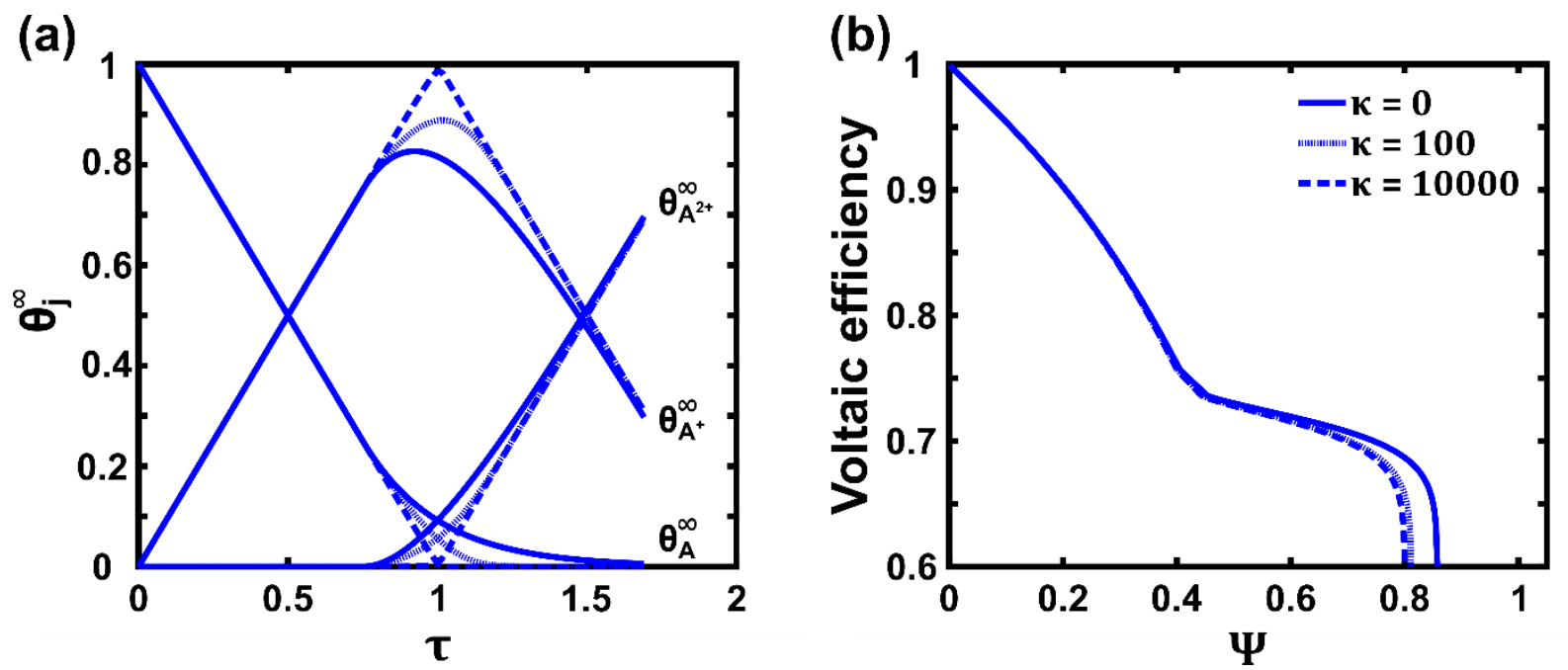

Figure 6. (a) Dimensionless concentration profiles as a function of dimensionless time $(\tau)$, shown for the first charging step ( $\Psi=0.25)$ and (b) voltaic efficiency as a function of dimensionless charging current $(\Psi)$ for varying values of the dimensionless comproportionation rate constant: 
$\kappa=0, \quad \kappa=100$, and $\kappa=10000$. Results shown for $\gamma_{A^{+}}=\gamma_{A^{2+}}=0.8, \quad E_{\text {avg }}^{(A)}=2 \mathrm{~V}$, and $\Delta E^{(A)}=0.6 \mathrm{~V}$

\subsection{Full cell analysis with two-electron compounds at both electrodes}

To this point, we have only considered two-electron compounds in a half-cell, holding the counter half-cell at a constant reference potential $(0 \mathrm{~V})$. However, the presence of two-electron reactions in both half-cells presents additional voltage losses. Here, we introduce a second redoxactive species, $B$, which undergoes analogous two-electron transfer according to Equations (38) and (39) (Figure 1c):

$$
\begin{gathered}
B \rightleftharpoons B^{+}+e^{-} \\
B^{+} \rightleftharpoons B^{2+}+e^{-}
\end{gathered}
$$

Species $B$ is subject to the same governing equations as species $A$, which are detailed in the SI. For simplicity, we once again neglect ohmic losses and assume that mass transfer rates for all species are equal, the latter of which implies comproportionation / disproportionation reactions can also be ignored. In addition, we impose $\Delta E^{(A)}=\Delta E^{(B)}=0.6 \mathrm{~V}$ and $E_{\text {avg }}^{(B)}=0 \mathrm{~V}$ for all analyses presented here. Note that half-cell reactions proceed in opposite directions - a positive, oxidizing current applied to species $A$ corresponds to a negative, reducing current applied to species $B$, and vice versa.

While one would correctly expect voltage losses to double in the case of a full cell if $A$ and $B$ were symmetric (i.e., equal concentrations, volumes, states of charge, transport properties, cell features), there are additional factors when this symmetry deviates that further hinder performance. In particular, we study charge imbalance (i.e., where the half-cells exist at different states of charge), which may result from self-discharge reactions occurring in the bulk or undesired side 
reactions (e.g., solvent or supporting salt decomposition, crossover-induced self-discharge, redox species decay). For one-electron compounds, this primarily impacts the accessible capacity because one half-cell becomes capacity-limiting during charge while the other is capacity-limiting during discharge.$^{42}$ For two-electron compounds, the effects of charge imbalance become more pronounced, significantly impacting the shape of the charge / discharge curves and the subsequent voltaic efficiency. ${ }^{10}$ To quantify charge imbalance between the two half-cells, we vary the initial dimensionless concentration of $A\left(\theta_{A}^{o}\right)$, assuming the initial dimensionless concentration of $B^{2+}$ is 1.

Figure 7a shows representative cycling profiles for the full cell potential at varying degrees of charge imbalance. Most notably, because each compound features a potential difference $\left(\Delta E^{(j)}\right)$, the difference between plateaus is doubled for the full cell. In the presence of charge imbalance, an intermediate plateau appears, corresponding to the disappearance of the first potential plateau for one half-cell. For example, during the charging step, species $A$ will be fully oxidized to $A^{+}$ before species $B^{2+}$ can be fully reduced to $B^{+}$, resulting in the intermediate plateau, which extends with increasing degrees of charge imbalance. Figure $\mathbf{7 b}$ shows the resultant effect of these behaviors on voltaic efficiency. Compared to the single half-cell examined in Figure 4, the inclusion of both half-cells doubles the associated losses and further diminishes the voltaic efficiency. Like the case of different mass transfer rates, charge imbalance leads to a characteristic region corresponding to the sequential disappearance of charge / discharge plateaus at increasing dimensionless currents. However, despite the reduction in dimensionless capacity shown in Figure 7a, charge imbalance causes only minor losses in voltaic efficiency at low to moderate currents $(0.2<\Psi<0.5)$. Overall, the combined effects of two-electron compounds at both electrodes and charge imbalance result in heightened voltaic efficiency losses compared to the single half-cell. 
(a)

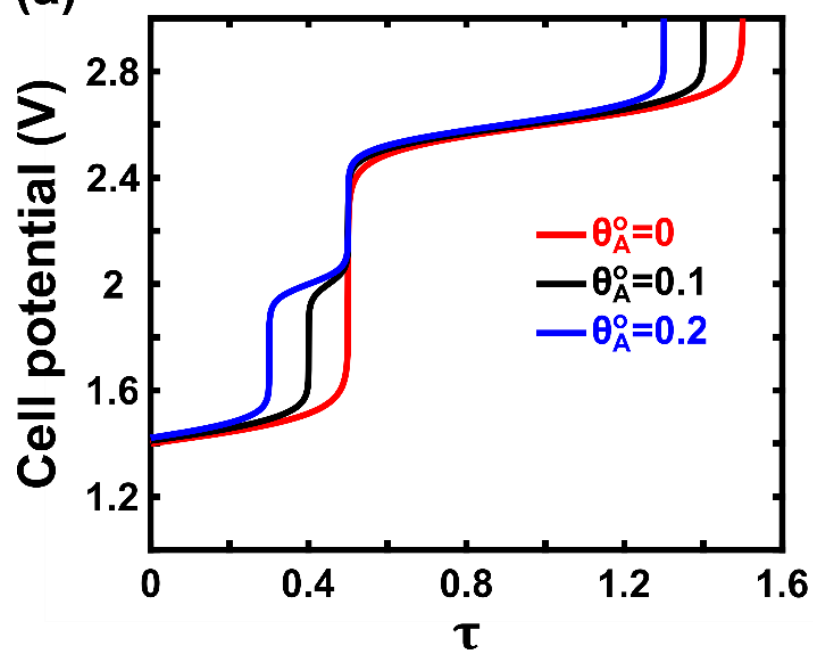

(b)

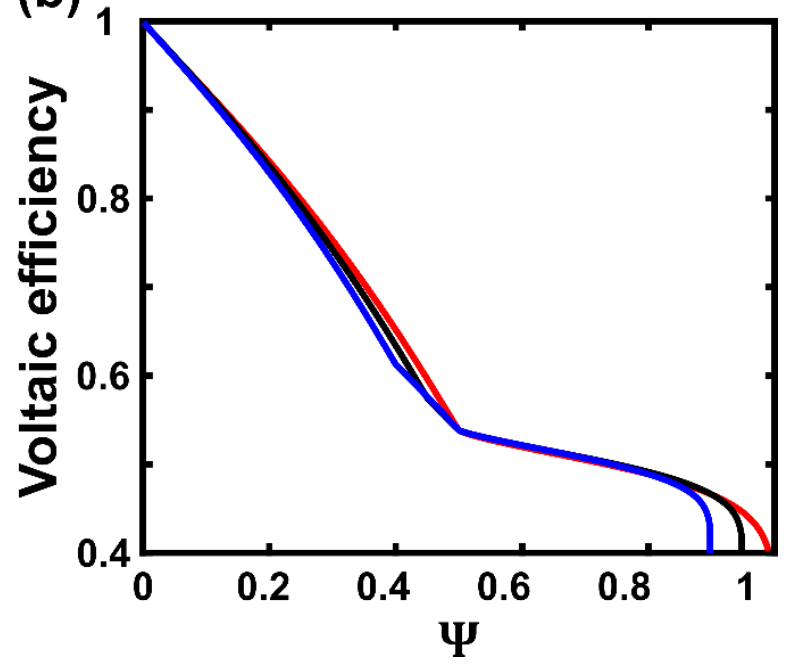

Figure 7. (a) Charge / discharge profiles shown for $\Psi=0.25$ and (b) voltaic efficiency as a function of dimensionless current for varying extents of charge imbalance. Results shown for $E_{\text {avg }}^{(A)}=2 \mathrm{~V}, E_{\text {avg }}^{(B)}=0 \mathrm{~V}, \Delta E^{(A)}=\Delta E^{(B)}=0.6 \mathrm{~V}, \gamma_{j}=1$, and $\kappa=0$.

\section{Design strategies for multi-electron RFBs}

The models explored here provide initial evidence that the multi-electron approach, while promising, has significant limitations if operating conditions and molecular design are not carefully considered. Even under optimistic conditions (i.e., losses at one electrode, reversible kinetics, no ohmic losses), we observe $10-20 \%$ losses in voltaic efficiency at moderate dimensionless currents ( $\Psi \approx 0.2$ ), and these losses grow as the average redox potential decreases and potential difference increases (ca. $30-40 \%$ losses). Therefore, the advantages of increased charge storage capacity in multi-electron transfer must be weighed against the drawbacks in flow battery performance for a given redox chemistry. This may restrict the viable operating space to applications where costs are less dependent on energy efficiency, as the ability to tolerate voltage losses is closely tied to techno-economics and system specifications. For example, long-duration and/or low current energy storage applications may be comparatively more tolerant of low voltaic 
efficiencies if other related capital costs are sufficiently low. ${ }^{43,44}$ Alternatively, higher cell voltages, enabled by non-aqueous electrolytes ${ }^{8}$ and certain aqueous electrolytes, ${ }^{45}$ can at least partially offset the increased overpotentials of multi-electron transfer (Figure 4).

To reduce the losses associated with sequential multi-electron transfer, molecular engineering efforts should aim to minimize the potential difference between electron transfer events without sacrificing cell voltage, solubility, or stability. Independently tuning the properties of individual redox events (e.g., potential inversion ${ }^{46}$ ) is a significant challenge, but future campaigns can build upon previously established examples and strategies, presenting new opportunities for research in this area ${ }^{4-51}$ For example, Kini et al. demonstrated that non-aqueous anthraquinone derivatives may undergo potential inversion by virtue of successive benzo substitution. Upon functionalization from the tetracyano napthaquinone to the analogous anthraquinone derivative, a shift is observed from sequential one-electron transfer events to a concerted two-electron transfer due to the distorted, non-planar molecular structure of the anthrquinone. ${ }^{47,50}$ This highlights the need for a more holistic conceptualization of the combined steric and electronic character of such molecules that influence their electrochemical nature, which should serve to advance fundamental knowledge in molecular electrochemistry and lead to improved non-aqueous RFB chemistries. Conversely, many aqueous organic compounds that undergo proton-coupled electron transfer already exhibit multi-electron reactions with a minimal potential difference, ${ }^{13,16,17}$ but these electron transfer reactions should be carefully scrutinized to elucidate gaps between their redox potentials, ${ }^{52}$ as the low cell voltages of these systems make them more susceptible to performance losses. 


\section{Conclusions}

The emergence of charge storage materials that support the reversible transfer of multiple electrons provides new pathways toward high energy density, low-cost charge storage. However, depending on the electrochemical and transport properties of the redox electrolyte, these multielectron compounds face inherent design tradeoffs between improved storage capacity and diminished voltaic efficiency. Here, we have explored various electrochemical models to describe the underlying phenomenology of two-electron transfer in RFBs and to estimate upper bounds for these performance tradeoffs, focusing on the redox electrolyte properties and foregoing more complex geometric constraints. These results reveal that the charging rate $(\Psi)$, average redox potential $\left(E_{\text {avg }}^{(j)}\right)$, and potential difference between redox events $\left(\Delta E^{(j)}\right)$ primarily drive voltaic efficiency losses, with relative mass transfer rates $\left(\gamma_{j}\right)$ and comproportionation rates $(\kappa)$ playing more nuanced roles. Considering the possible limitations, synthetic chemists and material scientists may seek new strategies to tune independent redox events, such as introducing concepts of potential inversion for non-aqueous electrolytes or leveraging existing knowledge of protoncoupled electron transfer for aqueous electrolytes. This low-dimensional modeling approach can further serve as a framework to bound performance for novel charge storage materials, including those with more complex electron transfer mechanisms, and to predict performance prior to embarking on complicated and time-consuming cell cycling studies. 


\section{CRediT authorship contribution statement}

Bertrand J. Neyhouse: Conceptualization, Methodology, Software, Validation, Investigation, Data curation, Visualization, Writing - original draft, Writing - review and editing. Alexis M. Fenton Jr.: Validation, Visualization, Writing - original draft, Writing - review and editing. Fikile R. Brushett: Conceptualization, Project administration, Supervision, Writing - original draft, Writing - review and editing.

\section{Acknowledgements}

This work was funded by the National Science Foundation (NSF) under Award Number 1805566. B.J.N. gratefully acknowledges the NSF Graduate Research Fellowship Program under Grant Number 1122374. Any opinion, findings, and conclusions or recommendations expressed in this material are those of the authors and do not necessarily reflect the views of the NSF. The authors also thank Katharine Greco, Dr. Jeffrey Kowalski, Kara Rodby, and Kevin Tenny of the Brushett Research Group for insightful discussion. 


\section{List of variables and constants}

$C_{j}^{z} \quad$ Concentration of species $j$ in state $z\left(\mathrm{~mol} \mathrm{~m}^{-3}\right)$

$C_{T}^{\infty} \quad$ Total bulk species concentration $\left(\mathrm{mol} \mathrm{m}^{-3}\right)$

$D \quad$ Diffusion coefficient $\left(\mathrm{m}^{2} \mathrm{~s}^{-1}\right)$

$E \quad$ Electrode potential (V)

$E_{1}^{(j)} \quad$ Formal redox potential for the first oxidation of $\operatorname{species} j(\mathrm{~V})$

$E_{2}^{(j)} \quad$ Formal redox potential for the second oxidation of species $j(\mathrm{~V})$

$E_{\text {avg }}^{(j)} \quad$ Average redox potential of species $j(\mathrm{~V})$

$\Delta E^{(j)} \quad$ Difference in formal redox potentials of species $j(\mathrm{~V})$

$F \quad$ Faraday's constant $\left(96485 \mathrm{C} \mathrm{mol}^{-1}\right)$

I Galvanostatic charging current (A)

$I_{1} \quad$ Partial current for the first oxidation (A)

$I_{2} \quad$ Partial current for the second oxidation (A)

$k_{d} \quad$ Disproportionation rate constant $\left(\mathrm{m}^{3} \mathrm{~mol}^{-1} \mathrm{~s}^{-1}\right)$

$k_{m, j} \quad$ Convective mass transfer coefficient of species $j\left(\mathrm{~m} \mathrm{~s}^{-1}\right)$

$k_{p} \quad$ Comproportionation rate constant $\left(\mathrm{m}^{3} \mathrm{~mol}^{-1} \mathrm{~s}^{-1}\right)$

$n \quad$ Number of electrons transferred per redox reaction

$N_{j} \quad$ Molar flux of species $j\left(\mathrm{~mol} \mathrm{~m}^{-2} \mathrm{~s}^{-1}\right)$

$r_{d} \quad$ Bulk disproportionation rate $\left(\mathrm{mol} \mathrm{m}^{-3} \mathrm{~s}^{-1}\right)$

$r_{p} \quad$ Bulk comproportionation rate $\left(\mathrm{mol} \mathrm{m}^{-3} \mathrm{~s}^{-1}\right)$

$R \quad$ Universal gas constant $\left(8.314 \mathrm{~J} \mathrm{~mol}^{-1} \mathrm{~K}^{-1}\right)$

$S \quad$ Electrode surface area $\left(\mathrm{m}^{2}\right)$

$t \quad$ Time (s)

$t_{\text {sand }}^{(n)} \quad$ Sand's time for a redox reaction with $n$ electrons (s)

$T \quad$ Temperature (K)

$V \quad$ Electrolyte volume $\left(\mathrm{m}^{3}\right)$

$x \quad$ Axial position perpendicular to the planar electrode surface (m)

\section{Greek symbols}

$\gamma_{j} \quad$ Dimensionless mass transfer coefficient of species $j$

$\eta \quad$ Integration variable (s)

$\theta_{j}^{z} \quad$ Dimensionless concentration of species $j$ in state $z$

$\kappa$ Dimensionless comproportionation rate

$\tau \quad$ Dimensionless charging time

$\Psi \quad$ Dimensionless charging current 


\section{Superscripts}

$s \quad$ Surface

$\infty \quad$ Bulk solution

$o \quad$ Initial state 


\section{References}

1. M. L. Perry and A. Z. Weber, J. Electrochem. Soc., 163, A5064-A5067 (2016).

2. D. G. Kwabi, Y. Ji, and M. J. Aziz, Chem. Rev., 120, 6467-6489 (2020).

3. F. R. Brushett, M. J. Aziz, and K. E. Rodby, ACS Energy Lett., 5, 879-884 (2020).

4. J. A. Kowalski, L. Su, J. D. Milshtein, and F. R. Brushett, Curr. Opin. Chem. Eng., 13, 45-52 (2016).

5. R. W. Hogue and K. E. Toghill, Curr. Opin. Electrochem., 18, 37-45 (2019).

6. R. Chen, Curr. Opin. Electrochem., 21, 40-45 (2020).

7. M. Li, Z. Rhodes, J. R. Cabrera-Pardo, and S. D. Minteer, Sustain. Energy Fuels, 4, 43704389 (2020).

8. Y. Yan, S. G. Robinson, M. S. Sigman, and M. S. Sanford, J. Am. Chem. Soc., 141, 1530115306 (2019).

9. J. A. Kowalski, M. D. Casselman, A. P. Kaur, J. D. Milshtein, C. F. Elliott, S. Modekrutti, N. H. Attanayake, N. Zhang, S. R. Parkin, C. Risko, F. R. Brushett, and S. A. Odom, J. Mater.

Chem. A, 5, 24371-24379 (2017).

10. N. H. Attanayake, J. A. Kowalski, K. V. Greco, M. D. Casselman, J. D. Milshtein, S. J.

Chapman, S. R. Parkin, F. R. Brushett, and S. A. Odom, Chem. Mater., 31, 4353-4363 (2019).

11. C. S. Sevov, S. L. Fisher, L. T. Thompson, and M. S. Sanford, J. Am. Chem. Soc., 138, 15378-15384 (2016).

12. K. H. Hendriks, C. S. Sevov, M. E. Cook, and M. S. Sanford, ACS Energy Lett., 2, 24302435 (2017).

13. B. Yang, L. Hoober-Burkhardt, S. Krishnamoorthy, A. Murali, G. K. S. Prakash, and S. R. Narayanan, J. Electrochem. Soc., 163, A1442-A1449 (2016).

14. L. Tong, M.-A. Goulet, D. P. Tabor, E. F. Kerr, D. De Porcellinis, E. M. Fell, A. AspuruGuzik, R. G. Gordon, and M. J. Aziz, ACS Energy Lett., 4, 1880-1887 (2019).

15. B. Hu, J. Luo, M. Hu, B. Yuan, and T. L. Liu, Angew. Chem., 131, 16782-16789 (2019).

16. C. Zhang, Z. Niu, S. Peng, Y. Ding, L. Zhang, X. Guo, Y. Zhao, and G. Yu, Adv. Mater., 31, 1901052 (2019).

17. C. Wang, X. Li, B. Yu, Y. Wang, Z. Yang, H. Wang, H. Lin, J. Ma, G. Li, and Z. Jin, ACS Energy Lett., 5, 411-417 (2020).

18. M. Quan, D. Sanchez, M. F. Wasylkiw, and D. K. Smith, J. Am. Chem. Soc., 129, 1284712856 (2007). 
19. G. Kwon, S. Lee, J. Hwang, H.-S. Shim, B. Lee, M. H. Lee, Y. Ko, S.-K. Jung, K. Ku, J. Hong, and K. Kang, Joule, 2, 1771-1782 (2018).

20. B. Hu and T. L. Liu, J. Energy Chem., 27, 1326-1332 (2018).

21. J. Chai, A. Lashgari, X. Wang, and J. “Jimmy” Jiang, J. Electrochem. Soc., 167, 100556 (2020).

22. T. Liu, X. Wei, Z. Nie, V. Sprenkle, and W. Wang, Adv. Energy Mater., 6, 1501449 (2016).

23. C. DeBruler, B. Hu, J. Moss, J. Luo, and T. L. Liu, ACS Energy Lett., 3, 663-668 (2018).

24. J. Luo, B. Hu, C. Debruler, and T. L. Liu, Angew. Chem. Int. Ed., 57, 231-235 (2018).

25. P. J. Cabrera, X. Yang, J. A. Suttil, K. L. Hawthorne, R. E. M. Brooner, M. S. Sanford, and L. T. Thompson, J. Phys. Chem. C, 119, 15882-15889 (2015).

26. S. M. Laramie, J. D. Milshtein, T. M. Breault, F. R. Brushett, and L. T. Thompson, J. Power Sources, 327, 681-692 (2016).

27. B. Kumar Chakrabarti, E. Kalamaras, A. Kumar Singh, A. Bertei, J. Rubio-Garcia, V. Yufit, K. M. Tenny, B. Wu, F. Tariq, Y. S. Hajimolana, N. P. Brandon, C. T. J. Low, E. P. L. Roberts, Y.-M. Chiang, and F. R. Brushett, Sustain. Energy Fuels, 4, 5433-5468 (2020).

28. M. Pugach, M. Kondratenko, S. Briola, and A. Bischi, Appl. Energy, 226, 560-569 (2018).

29. S. B. Lee, K. Mitra, H. D. Pratt, T. M. Anderson, V. Ramadesigan, B. R. Chalamala, and V. R. Subramanian, J. Electrochem. Energy Convers. Storage, 17 (2020).

30. S. B. Lee, H. D. Pratt, T. M. Anderson, K. Mitra, B. R. Chalamala, and V. R. Subramanian, ECS Trans., 85, 43 (2018).

31. A. Tang, J. Bao, and M. Skyllas-Kazacos, J. Power Sources, 196, 10737-10747 (2011).

32. S. V. Modak and D. G. Kwabi, ECS Meet. Abstr., MA2020-02, 2670 (2020).

33. J.-M. Savéant and C. Costentin, Elements of Molecular and Biomolecular Electrochemistry: An Electrochemical Approach to Electron Transfer Chemistry, p. 640, John Wiley \& Sons, (2019).

34. A. J. Bard and L. R. Faulkner, Electrochemical Methods: Fundamentals and Applications, Wiley, (2000).

35. R. Darling, K. Gallagher, W. Xie, L. Su, and F. Brushett, J. Electrochem. Soc., 163, A5029A5040 (2016).

36. R. M. Darling, K. G. Gallagher, J. A. Kowalski, S. Ha, and F. R. Brushett, Energy Environ. Sci., 7, 3459-3477 (2014). 
37. H. Wang, S. Y. Sayed, E. J. Luber, B. C. Olsen, S. M. Shirurkar, S. Venkatakrishnan, U. M. Tefashe, A. K. Farquhar, E. S. Smotkin, R. L. McCreery, and J. M. Buriak, ACS Nano, 14, 25752584 (2020).

38. K. V. Greco, A. Forner-Cuenca, A. Mularczyk, J. Eller, and F. R. Brushett, ACS Appl. Mater. Interfaces, 10, 44430-44442 (2018).

39. J. L. Barton, J. D. Milshtein, J. J. Hinricher, and F. R. Brushett, J. Power Sources, 399, 133143 (2018).

40. D. H. Evans, Chem. Rev., 108, 2113-2144 (2008).

41. M. W. Lehmann and D. H. Evans, Anal. Chem., 71, 1947-1950 (1999).

42. M.-A. Goulet and M. J. Aziz, J. Electrochem. Soc., 165, A1466 (2018).

43. Z. Li, M. S. Pan, L. Su, P.-C. Tsai, A. F. Badel, J. M. Valle, S. L. Eiler, K. Xiang, F. R. Brushett, and Y.-M. Chiang, Joule, 1, 306-327 (2017).

44. P. Albertus, J. S. Manser, and S. Litzelman, Joule, 4, 21-32 (2020).

45. B. H. Robb, J. M. Farrell, and M. P. Marshak, Joule, 3, 2503-2512 (2019).

46. D. H. Evans and K. Hu, J. Chem. Soc. Faraday Trans., 92, 3983-3990 (1996).

47. A. M. Kini, D. O. Cowan, F. Gerson, and R. Moeckel, J. Am. Chem. Soc., 107, 556-562 (1985).

48. M. W. Lehmann, P. Singh, and D. H. Evans, J. Electroanal. Chem., 549, 137-143 (2003).

49. M. D. Sampson, A. D. Nguyen, K. A. Grice, C. E. Moore, A. L. Rheingold, and C. P. Kubiak, J. Am. Chem. Soc., 136, 5460-5471 (2014).

50. D. E. Wellman and R. West, J. Am. Chem. Soc., 106, 355-360 (1984).

51. K. Hernández-Burgos, G. G. Rodríguez-Calero, W. Zhou, S. E. Burkhardt, and H. D. Abruña, J. Am. Chem. Soc., 135, 14532-14535 (2013).

52. E. W. Zhao, T. Liu, E. Jónsson, J. Lee, I. Temprano, R. B. Jethwa, A. Wang, H. Smith, J. Carretero-González, Q. Song, and C. P. Grey, Nature, 579, 224-228 (2020). 